\title{
Application of OPC in the Intelligent Building System Integration
}

\author{
Jie Chen \\ Dongying Vocational College \\ Dongying 257091, P.R.China
}

\author{
Xiuli Sun \\ The control system education of Jinan university \\ Jinan 250022, P.R.China
}

\author{
Ruiyuan Jia \\ Dongying municipal commission of housing and urban-rural development, \\ Dongying 257091, P.R.China
}

\begin{abstract}
System integration is to integrate every weak current subsystem in the building on a computer network platform to realize sharing of information, resource and task, this platform can receive or transmit data and information of subsystem by network, serial port and data base to achieve control of every subsystem. This paper presents a method to realize intelligent building information integration based on OPC, and gives the concrete realization of OPC servers and OPC client sides combined with the weak current system of Dongsheng building.
\end{abstract}

\section{Keywords-OPC, weak current, system integration}

\section{INTRODUCTION}

Intelligent building light-current systems make the computer technology, the network technology, modern control technology, graphical display technology and communication technology an organic whole. It is the extremely important constituent part of intelligent building and it supervises all electrical equipment of the whole building. Light-current systems generally include building automation system (BAS), fire-fighting automation alarm subsystem (FAS), security subsystem (SAS), satellite and cable TV subsystem (CATV), garage management subsystem (CPS), public broadcasting and emergency broadcast subsystem (PAS). Light-current system's integration integrates each subsystem into a flat to realize linkage control and information sharing, and complete the management of an entire building.

To realize the system integration of intelligent buildings, we can use OPC technology to solve the communication question of integrated management platform and site equipment and subsystems. The emergence of this technology offers a full set of solutions for the comprehensive solvement of various different manufacturer's all kinds of equipment and system integration.

\section{OPC TECHNOLOGY}

Definition of OPC

OPC is a communication standard based on OLE. It includes a set of interfaces, a standard set of properties and methods for process control and manufacturing automation systems.

OPC focused on solving the standardization of reading and writing data and data transmission between the application software and process control equipment etc. It followed the Client / Server model, regarding the data collection terminal as OPC Server, and the other side as OPC Client access.

OPC provides information management domain application software and real-time control domain for data transmission, and provides data from control equipment of access process of application software, solving standards problem of communication between the application software and process control equipment. When the device is connected through OPC, drivers of field devices such as the graphics application software, application software of trend analysis and alarm applications software and so on, are based on the OPC standard. In unified OPC environment, the application can read directly data from field devices, without compiling special procedures for interface separately, and each field device can be interconnected directly with the different applications. Improvement and extension of OPC technology created a good software environment, for integration of the intelligent building system, especially comprehensive integration of the real-time control domain and information management domain.

As the middle layer of the integrated system, OPC server will connect control signal with management software in accordance with uniform standards seamlessly, and separate the hardware and software applications effectively. OPC server program by calling the hardware drivers to access the dual-port RAN in intelligent network adapter, and applications, through the OPC interface with OPC server, exchange data, to indirectly achieve on-site information.

Based on DCOM technology, OPC technology effectively supports communication between distributed applications in network. This will be monitoring computer via an Ethernet and other computers to make up LAN, and client programs distributed in other computer can communicate with OPC servers of control computers, to achieve information sharing, which will undoubtedly promote the integration and fusion of each subsystem. Intelligent Building of each subsystem, through OPC servers, achieves data exchange in different field devices. Intelligent systems of the whole building monitor and manage in a platform, to realize flexible configuration of the building intelligent system integration and multisystem management. 

system

Application model of OPC in the building intelligent

OPC technology is applied to the intelligent building system integration, application model block diagram shown in Figure 1.

In the above model, the integrated platform collects data of this system, deals with communication between the various subsystems, and provides a centralized decision-making and control. These functions are completed by all objects of the integration platform, and the conceptual model is divided into three layers: 1) the data communication layer: composed of the BAS client interface, the CAS client interface and OAS client interface, and it uses the standard OPC interface and the Subsystem objects interact ,to complete the acquisition of status of each subsystem, the log, switch signals, etc.; 2) the control layer of analysis: divided into database object management, log management objects, and system management objects, system state management objects and so on, and it analyzes, sorts and filters data gained, generating reports, logs, and control signals; 3) the assisted decision-making layer: comprised of the object of the joint management, the user interface and supporting decision-making etc, and it can provide assisted decision-making abilities on the basis of data analysis.

Subsystem object is an important annulus of an integrated model, and the main function is packaging the subsystem status and alarm information, using formats defined of standard information and standard interfaces to communicate with the integration platform; receiving control information from the platform, through calling the file from subsystem, database and application system, to complete control, while avoiding increasing system burden field devices direct access to integration platform.

Between the operating station of the subsystem and field equipment, the various subsystems, through the OPC user interface, interact data with field equipment with server interface of standard OPC, and integrated management platform, through the OPC client interface, interacts data with subsystems, to achieve the purpose of control and management. If you need some special field devices to communicate directly with the IBMS, you can access directly through OPC interfaces to the IBMS without the subsystem level. Thus, if subsystems or field devices meet the OPC specifications, they can access the system. Customer can choose different manufacturers according to their need. When a subsystem or field device needs to modify or upgrade, it just modifies the corresponding OPC server interface or OPC client interface, without involving integrated management platform, to shield the heterogeneity of the subsystems, The openness and maintainability of the system effectively enhanced.

\section{THE APPLICATION OF OPC IN INTEGRATION OF INTELLIGENT BUILDING SYSTEM}

Dongsheng Mansion, located in Xicheng, Dongying, area of $30 \mathrm{mu}$, floor space of 51000 square meters, the height of 99.7 maters, with 26 floors on the ground and 2 floors under the ground, the outdoor patio area of about 22,000 square meters, is an integrated intelligent building with hotels, business, entertainment and restaurants. To achieve these objectives, we need an integrated system, to integrate the information exchange of sub-systems of the intelligent systems, to achieve the centralized monitoring and control of information about a system, and to achieve the linkage of necessary and definable events between the various subsystems.

For the characteristics of Dongsheng Mansion, we propose a solution based on OPC, Floor control system is the basis of the system integration, the building control management system as a platform of system-wide integration, the OPC as an information exchange platform, every system as an OPC server, and then integrated to the final OPC client. Integration of the system architecture into three layers: The first layer is the integration of subsystems, Such as the integration between burglar alarm and CCTV, then set the OPC server on the basis of the first layer, forms a second layer of integration, OPC server to the information management center as the third level integration.

The components of light-current system.

The light-current system of Dongsheng Mansion involves background music; emergency broadcast system, automatic fire alarm system, CCTV surveillance system, anti-theft alarm system and Access control system. These systems integrate at the basis of Siemens's $\$ 600$, OPC as the main actualize way, apart from this, each subsystem has done some more closely control linkage according to functional needs, horizontal integration in other words, such as the control linkage of background music and emergency radio subsystem, fire alarm and linkage control system, CCTV surveillance system, the integration Anti-theft alarm system and access control system, the Integration of OPC Server Building Automation Systems. The below describe is in the example of integration of the building automation system and OPC server.

The automation system of Dongsheng Mansion includes: (1) Monitoring of HVAC systems; (2) Monitoring of Water supply and drainage system; (3)Monitoring of Power supply and lighting system; (4) Elevator control. Floor control system is the basis for the system integration, the building control management system as the platform of system-wide integration, the OPC as an information exchange platform, each system as an OPC server, the final integrated as the OPC client. In the other words, the Integration of the system architecture includes three layers: The first layer is the integration of sub-systems, such as Such as the integration between burglar alarm and CCTV, then set the OPC server on the basis of the first layer, form a second layer of integration, OPC server to the information management center as the third level integration.

In order to achieve three integrations above, in addition to using commercial OPC architecture, also need the self-programming of the first and the second layers. The following piece of code is the sending code of OPC server when alarm occurs.

CEventServer pEventServer $=$ 
((CAlarmDoc )GetDocument0)->GetEventServer0; if(pEventServer!=NULL)

$$
\}
$$$$
\{
$$$$
\text { if(pEventServer->ChooseServer0) }
$$$$
\{
$$

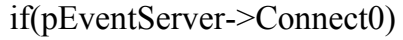$$
\{
$$$$
\text { / / settitle }
$$$$
\text { GetDocument } 0 \rightarrow \text { SetTitle }
$$$$
\text { (pEventServer->GetOPCServerName } 0 \text { ); }
$$

The realization of light-current system integration of Dongsheng Mansion.

Systems Dongsheng Mansion has integrated include: building automation system, fire-fighting automation alarm system, television monitoring system, smart cards system, intelligent fire alarm system and patrol management system and so on.

Firstly, link each subsystem above together by intelligent interface and data gateway, central integrated management system exchange data by Ethernet and data gateway, data gateway being a client side of central integrated management system, client side transmits data to server by means of immediacy and timing. Immediate transmit is that once alarm information occurs, it should be transmitted to central integrated management system so that central integrated management system can linkage other subsystem. Intelligent interface transforms information transmitted from each subsystem to layout needed by central integrated management system, then transmit to data gateway, finally, data gateway packs it and transmit it in communication protocol which is decided in advance.

Data gateway provides a unique port for central integrated management system which reduces the burdens of central integrated management system and is convenient for system maintenance and successful integration as a client side of OPC Server communicating with central integrated management system, serving the function of information transfer among each subsystem, and is the pivot of the whole information transmits. It manages each IEI, distinguish and receive each IEI information, for instance, the information from IEI needs immediate transmit or timing transmit, then packs packet OPC can distinguish and transmit it.

IEI can accomplish transfer of date layout and communication protocol, and hardware drivers of different hardware interface such as standard RS-232 interface, difference RS-485, or RS-422 interface, serial printer interface and so on. It transforms different command format of each subsystem so that it is convenient for central integrated management system to receive distinguish and manage. Integrated schematic is as follows:

In the way above, the integration of building automation systems, burglar alarm system, TV monitoring systems, smart card systems, fire alarm systems, radio systems, patrol management system into a platform was realized finally. According to their importance, Integrate gradational various isolated devices, features and information into an associated integrated, unified and coordinated system that allows full sharing of resources, efficient management, easily use. The sharing of the system information indicates that the bridge between any two subsystems has been established, Therefore, the linkage problem between the various subsystems solved. Here are two typical integrated joint actions to manifest it:

The anti-theft alarm system and the CCTV monitoring system cooperate with the Building Automation System. While the anti-theft alarm signal generates, cameras of the CCTV monitoring system will be rechecked and take video. If it's not bright enough, the Building Automation System will turn on the lights in the alarm zone immediately. The cooperation of the anti-theft alarm system and the CCTV monitoring system is realized by the Sharing Alarm. And then their integration with the Building Automation System is achieved by the Sharing Alarm via the integration of database.

While the cooperation of the Smart Card System and the CCTV monitoring system is a little different. If something wrong happens when someone put the card on the Card Reader, the Building Automation System will deliver an alarm signal to the Sharing Alarm through the OPC Server and the Sharing Alarm will give orders to the CCTV monitoring system to switch the video screen according to the coding of the alarm signal. Then the CCTV monitoring system controls the matrix to switch the cameras in order to make near confirmation and take video.

\section{Discussion}

The system integration of intelligent building is not simple accumulation of every subsystem, but organic integration of each subsystem functions. The key of the organic integration is how to design and structure. This article applies OPC technology on the design of intelligent building system integration so as to realize the open and seamless connection of every subsystem. This method save manpower, money, and improve the response ability of the building to unforeseen events. 


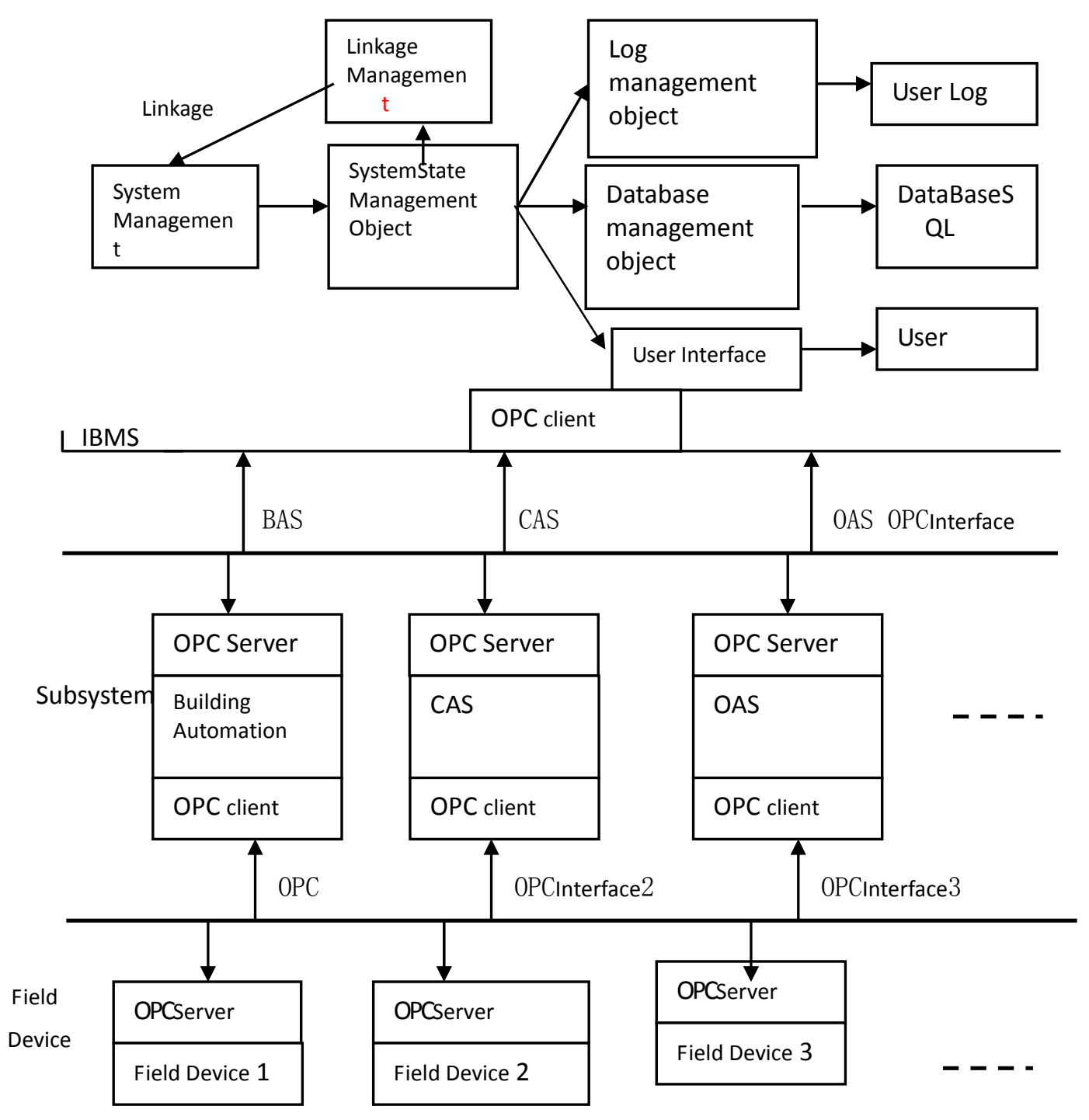

Figure 1. Application model diagram 


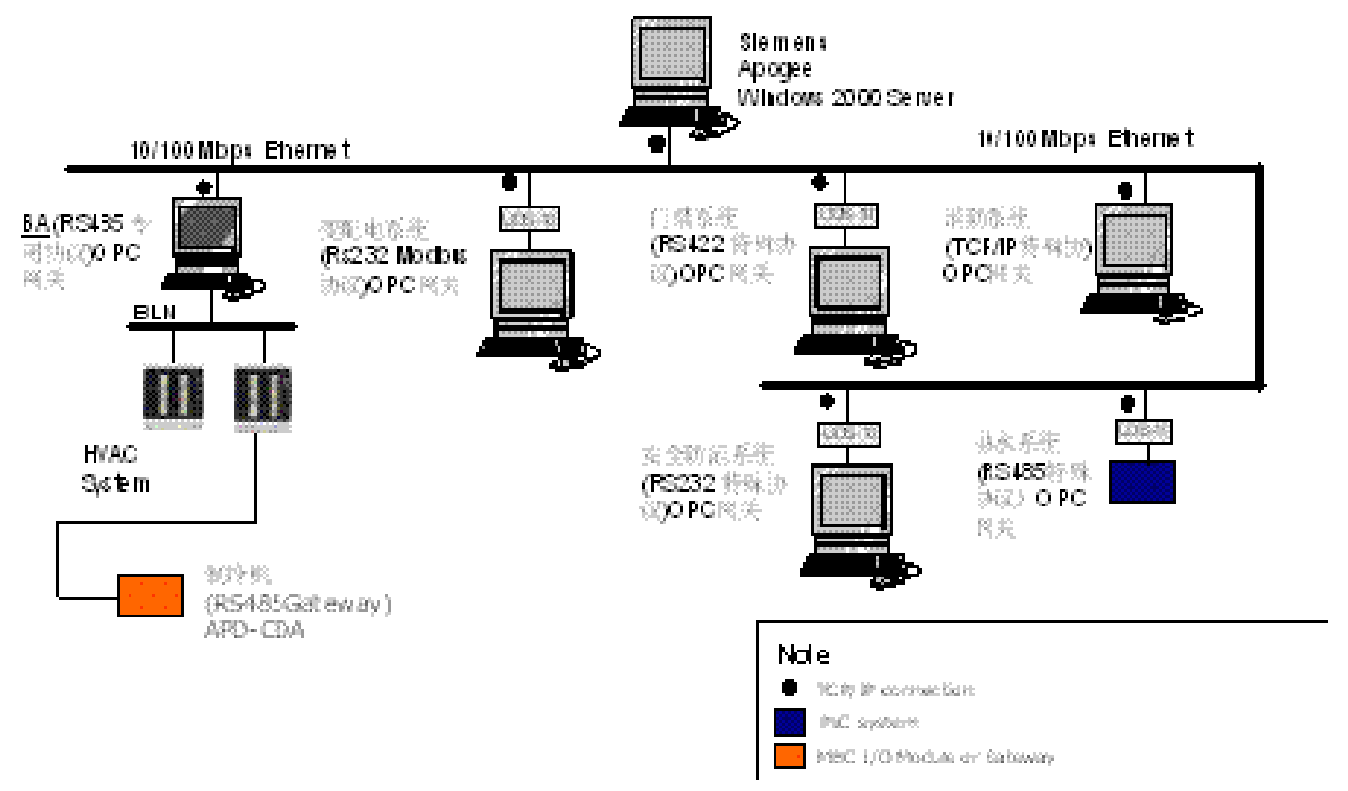

Figure 2. The integrated schematic of the systems of Dongsheng building 